\title{
Estimating subthreshold tumor on MRI using a 3D-DTI growth model for GBM: An adjunct to radiation therapy planning
}

\author{
LEITH HATHOUT $^{1}$ and VISHAL PATEL ${ }^{2}$ \\ ${ }^{1}$ Harvard Medical School, Boston, MA 02115-5750; ${ }^{2}$ Department of Radiological Sciences, \\ David Geffen School of Medicine, University of California-Los Angeles, Los Angeles, CA 90095, USA
}

Received March 17, 2016; Accepted May 11, 2016

DOI: $10.3892 /$ or.2016.4878

\begin{abstract}
Mathematical modeling and serial magnetic resonance imaging (MRI) used to calculate patient-specific rates of tumor diffusion, $D$, and proliferation, $\rho$, can be combined to simulate glioblastoma multiforme (GBM) growth. We showed that the proportion and distribution of tumor cells below the MRI threshold are determined by the $D / \rho$ ratio of the tumor. As most radiation fields incorporate a $1-3 \mathrm{~cm}$ margin to account for subthreshold tumor, accurate characterization of subthreshold tumor aids the design of optimal radiation fields. This study compared two models: a standard one-dimensional (1D) isotropic model and a three-dimensional (3D) anisotropic model using the advanced imaging method of diffusion tensor imaging (DTI) - with regards to the $D / \rho$ ratio's effect on the proportion and spatial extent of the subthreshold tumor. A validated reaction-diffusion equation accounting for tumor diffusion and proliferation modeled tumor concentration in time and space. For the isotropic and anisotropic models, nine tumors with different $D / \rho$ ratios were grown to a $\mathrm{T} 1$ radius of $1.5 \mathrm{~cm}$. For each tumor, the percent and extent of tumor cells beyond the $\mathrm{T} 2$ radius were calculated. For both models, higher $D / \rho$ ratios were correlated with a greater proportion and extent of subthreshold tumor. Anisotropic modeling demonstrated a higher proportion and extent of subthreshold tumor than predicted by the isotropic modeling. Because the quantity and distribution of subthreshold tumor depended on the $D / \rho$ ratio, this ratio should influence radiation field demarcation. Furthermore, the use of DTI data to account for anisotropic tumor growth allows for more refined characterization of the subthreshold tumor based on the patient-specific $D / \rho$ ratio.
\end{abstract}

Correspondence to: Dr Leith Hathout, Harvard Medical School, 107 Avenue Louis Pasteur, Boston, MA 02115-5750, USA

E-mail: leith_hathout@hms.harvard.edu

Key words: glioblastoma multiforme, mathematical modeling, diffusion tensor imaging, anisotropy, invisibility index

\section{Introduction}

Glioblastoma multiforme (GBM) represents the most common as well as most aggressive primary brain tumor, with a mean survival time of $12-14$ months following diagnosis (1-4). The highly proliferative and invasive nature of GBM leads to rapid tumor growth and poor prognosis despite treatment with a combination of surgery, radiotherapy, and chemotherapy. In treating GBM, the presumption of a clinically significant proportion of tumor cells below the imaging threshold of standard modalities such as magnetic resonance imaging (MRI) drives the clinical practice of incorporating large margins in the design of radiation fields. The inherent trade-off in establishing such large treatment margins is the increased radiation of normal brain tissue. Just as notable as the magnitude of these margins is the wide range of clinically acceptable margins. For example, radiation fields typically include a $1-3 \mathrm{~cm}$ margin beyond the observable tumor boundaries on MRI (5). Thus, a patient-specific, quantitative approach to assessing the proportion and extent of tumor cells beyond the $\mathrm{T} 2$ imaging threshold may provide insight into the optimal size and shape of radiation fields on an individual patient basis, ultimately leading to improved outcomes. One approach to individually tailored quantitative analysis of subthreshold tumor relies on the combination of standard MRI imaging techniques with mathematical modeling of GBM growth (6-9). According to the assumptions of the model, serial MR imaging can be used to calculate patient-specific rates of tumor cell diffusion and proliferation, termed $D$ and $\rho$, respectively $(8,10)$. These rates are then incorporated into the reaction-diffusion partial differential equation model of glioblastoma growth, allowing for individualized simulation of tumor growth and calculation of tumor cell concentrations as a function of time as well as space.

This model has been used in a variety of applications, including modeling the effects of surgery, chemotherapy and radiation therapy $(7,11-13)$. It has also been used to suggest a tumor classification based on individual values of $D$ and $\rho$ (14). This report focuses on the relative effects of diffusion and proliferation, i.e., the $D / \rho$ ratio, which can be shown to dictate the spatial distribution of tumor cells at any given time $(8,10)$. In this study, we demonstrated how the $D / \rho$ ratio, which may also be referred to as the 'invisibility index' of the tumor, can 
be used to predict the percent and extent of tumor cells beyond the T2 threshold, i.e., beyond the visible tumor margins on traditional clinical MRI sequences. These predictions can, in turn, be used as a tool for more optimal design of radiation fields (or surgical resection planning).

Traditional applications of the reaction-diffusion model have simulated glioblastoma growth in one spatial dimension and conceive of the patient-specific rate of diffusion, $D$, as a single value applicable throughout the brain, thereby modeling isotropic tumor growth $(6-8,12,13)$. Based on the finding that glioma cells diffuse more quickly through white matter than gray matter, a refinement of the model by Swanson et al used Brain Atlas Data to incorporate preferential white matter diffusion into the reaction-diffusion tumor model, with $D$ now being a spatially varying parameter $D(x)$, which would take different values in gray matter $D_{g}$ and white matter $D_{w}$, with $D_{w}$ being 5-100 times greater than $D_{g}(15)$. However, even in white matter, it is known that diffusion is not isotropic, and that tumor cells migrate preferentially in the direction of white matter tracts (16-20). Therefore, a uniform $D_{w}$ parameter is not strictly adequate.

Thus, the most recent research study has begun to incorporate diffusion tensor imaging (DTI) data into models of glioma growth, allowing for modeling anisotropic tumor spread (21). DTI represents an important advancement in tumor growth modeling because it allows for three-dimensional (3D) tumor modeling in the anatomical context of the brain. Specifically, the advent of diffusion tensor MR imaging has provided the capacity to map main fiber tracts, and provide directionality to the diffusion coefficient based on a diffusion tensor associated with each voxel. Therefore, the next step in model refinement was the incorporation of DTI data to allow a more realistic anisotropic diffusion of tumor cells and their preferential migration along fiber tracts (21), with $D$ now being a tensor incorporating the magnitude and directionality of the diffusion coefficient. This has been shown to enhance the accuracy of predicted tumor growth modeling (21). This more anatomically nuanced approach to GBM modeling has the potential to allow for more accurate assessment of the subthreshold tumor and, therefore, more optimal planning of radiation fields. Thus, the current report combines routine MR patient data to estimate bulk values of $D$ and $\rho$ and DTI data to refine directionality of simulated tumor growth. Results of this DTI-based anisotropic model are compared to that of the traditional one-dimensional (1D), isotropic simulation of GBM growth with regards to the effect of the $D / \rho$ ratio on the percent and spatial extent of tumor cells beyond the visible T2 boundary. Furthermore, we extended this analysis to consider how, for a given $D / \rho$ ratio, the initial location of tumor cells can lead to different tumor growth patterns in the anisotropic growth model and how this, in turn, affects the quantity and distribution of subthreshold tumor.

\section{Materials and methods}

Mathematical model. The mathematical model of glioblastoma growth employs a reaction-diffusion partial differential equation to calculate tumor cell density as a function of both time and space. Conceptually, the model relies on a mass-balance approach, such that the number of tumor cells in any given location can increase only by the local proliferation of tumor cells or by the migration of tumor cells into that location. Thus, the model accounts for both the infiltrative nature of the tumor using a diffusion term as well as the proliferation of tumor cells using a proliferation term. The proliferation of the tumor is typically modeled using a logistic growth term, such that the rate of proliferation in any given location decreases as the tumor cell density approaches the tissue carrying capacity (6).

In words, the tumor model can be stated as follows: Rate of change of tumor cell density (at a location $x$ ) = Net invasion (diffusion) of tumor cells + Net proliferation of tumor cells. Traditionally, this equation has been used to model isotropic diffusion of tumor cells in one spatial dimension. In such a context, there is a single, constant value of $D$ that is applicable throughout the domain of the brain and so the model can be written in mathematical terms as the classical Fisher-Kolmogorov reaction-diffusion equation $(6,7,9)$ :

$$
\frac{\partial c}{d t}=D \frac{\partial^{2} c}{d x^{2}}+\rho c\left(1-\frac{c}{K}\right)
$$

where the various terms of the equation are defined as follows.

In this equation, $c(t, x)$ represents the tumor cell density, in units of cells $/ \mathrm{mm}^{3}$, which is a function of position $x$ and time $t$. $D$ is the diffusion term, in $\mathrm{mm}^{2} /$ day, which models local invasion of tumor cells. $\rho$ is the tumor proliferation rate in units of (/day), and $\mathrm{K}$ is the tissue tumor-carrying capacity in units of cells $/ \mathrm{mm}^{3}$ and is typically set at $10^{5}$ cells $/ \mathrm{mm}^{3}(22)$.

However, the assumption of a single value for diffusion coefficient applicable throughout the brain, in other words that the brain is an entirely homogenous mass, does not adequately account for the anatomical complexities of the brain. For example, it has been observed that tumor cells diffuse more quickly in white matter than in gray matter (15). Thus, a more accurate representation of the diffusion term of the model, as suggested by Swanson et al, defined $D$ as a spatially dependent function, $D(x)$, that has one value in gray matter, $D_{g}$, and a different value in white matter, $D_{w}$, as noted above. With this definition of $D$, the model can be rewritten as:

$$
\frac{\partial c(t, x)}{d t}=\nabla \bullet(D(x) \nabla c)+\rho c\left(1-\frac{c}{K}\right)
$$

In this form, the equation treats the brain as a heterogeneous mass in which there is a different rate of diffusion in white matter as opposed to gray matter. However, even this formulation of the model does not fully capture the anatomical nuances of the brain, such as the anisotropy of the brain allowing for greater rates of tumor cell diffusion along the direction of white matter tracts. In order to account for anisotropic diffusion, this report follows the method of Jbabdi et al, modeling 3D growth of GBM guided by a cell diffusion tensor derived from the water diffusion tensor as given by MRI DTI imaging. Thus, the model can be rewritten as (21):

$$
\frac{\partial c(t, x)}{d t}=\nabla \bullet(\bar{D}(x) \nabla c)+\rho c\left(1-\frac{c}{K}\right)
$$

where $\bar{D}(x)$ is the $3 \times 3$ diffusion tensor that describes anisotropic diffusion as a function of location. This diffusion tensor is derived from the standard water diffusion tensor: 


$$
\bar{D}_{w}=\left(\begin{array}{ccc}
D_{x x} & D_{x y} & D_{x z} \\
D_{y x} & D_{y y} & D_{y z} \\
D_{z x} & D_{z y} & D_{z z}
\end{array}\right) .
$$

If we let $\left(\lambda_{i}, e_{i}\right)_{i=1,2,3}$ correspond to the eigenvectors and eigenvalues of $\bar{D}_{w}$, then $\bar{D}(x)$ can be written as follows: $\bar{D}(x)=\bar{\lambda}_{1}(r) e_{1} e_{1}^{T}+\bar{\lambda}_{2}(r) e_{2} e_{2}^{T}+\bar{\lambda}_{3}(r) e_{3} e_{3}^{T}$, where $\bar{\lambda}_{i}(r)$ modifies the eigenvalues of the diffusion tensor to account for the fact that cellular migration along white matter tracts displays greater anisotropy than does water diffusion. The form of $\bar{\lambda}_{i}(r)$ is as per Jbabdi et al (21). In general, this is referred to as the 'stretch factor', and is taken to be 10 for the purposes of this report (21). The cellular diffusion coefficient in any particular direction $v$ (where $v$ is a unit vector) can then be written as $\bar{D}(v)=\bar{\lambda}_{I}(r)\left(v \bullet e_{1}\right)^{2}+\bar{\lambda}_{2}(r)\left(v \bullet e_{2}\right)^{2}+\bar{\lambda}_{3}(r)\left(v \bullet e_{3}\right)^{2}(23)$. This will be useful in discretization of the solution, to calculate diffusion coefficients from one voxel to its nearest neighbors.

Once $\bar{D}(x)$ has been calculated, the equation can be developed as follows, once again as per Jbabdi et al:

$$
\frac{\partial c(t, x)}{d t}=\nabla \bullet(\bar{D}(x) \nabla c)+\rho c\left(1-\frac{c}{K}\right)
$$

can be transformed into the following equation:

$$
\frac{\partial c(t, x)}{d t}=\sum_{i, j=1}^{3} \bar{D}_{i, j} \frac{\partial^{2} c}{\partial x_{i} \partial x_{j}}+\sum_{i=1}^{3} \tilde{\bar{D}}_{i} \frac{\partial c}{\partial x_{i}}+\rho c\left(1-\frac{c}{K}\right)
$$

where

$$
\widetilde{\bar{D}_{i}}=(\bar{D} \nabla)_{i}=\sum_{j=1}^{3} \frac{\partial \bar{D}_{i, j}}{\partial x_{j}}
$$

In other words $\tilde{\bar{D}}$ is derived from the tensor $\bar{D}$ by calculating its element by element gradient.

For any given initial condition $c(0, x)=c_{o}(x)$, and zero flux boundary conditions such that tumor cells are prohibited from migrating outside the domain of the brain $(\bar{D}(x) \nabla c) \bullet n=0$, this model can be used to evaluate tumor cell concentration as a function of both time and space. The initial condition used in this study is a tumor cell concentration of $0.8^{*} \mathrm{~K}$ confined to a single voxel.

The model is then solved using a forward finite differences approach, using $\Delta t$ as a time step and $(\Delta x, \Delta y, \Delta z)$ as space steps. If the cellular tumor concentration over the entire space at time $n \Delta t$ is represented as $C^{n}$, then it is possible to develop the forward differences method in the form:

$$
\frac{C^{n+1}-C^{n}}{\Delta t}=A C^{n}
$$

where $A$ is a large sparse matrix. The elements of $A$ are a function of $\bar{D}, \widetilde{D}, \rho$, and the discretization $(\Delta t, \Delta x, \Delta y, \Delta z)$. The precise form of the matrix is developed as per Jbabdi et al (21).

Simulations. In this study, we investigate the effect of varying the $D / \rho$ ratio on the percent and extent of tumor cells beyond the observable $\mathrm{T} 2$ boundaries for both the $1 \mathrm{D}$ isotropic growth model and the $3 \mathrm{D}$ anisotropic model. $D / \rho$ was chosen as the parameter of interest because this ratio can be shown to be the primary driver of tumor cell distribution. This is shown by non-dimensionalizing the Fisher-Kolmogorov reaction-diffu- sion equation by introducing the following non-dimensional parameters: $\bar{t}=\rho t, \bar{x}=x / L, \bar{c}=c / K, D^{*}=D /\left(\rho L^{2}\right)$, where $L$ is the length of the 1D domain for which numerical solutions to the reaction-diffusion partial differential equation will be calculated. Substituting these parameters into the Fisher-Kolmogorov equation yields the following non-dimensionalized formulation of the model:

$$
K \rho \frac{\partial \bar{c}}{d \bar{t}}=D^{*} L^{2} \rho \frac{K}{L^{2}} \frac{\partial^{2} \bar{c}}{d \bar{x}^{2}}+K p \bar{c}(1-\bar{c})
$$

which can be further simplified to give:

$$
\frac{\partial \bar{c}}{d \bar{t}}=D^{*} \frac{\partial^{2} \bar{c}}{d \bar{x}^{2}}+\bar{c}(1-\bar{c})
$$

In this form, it is evident that the equation, and therefore the description of tumor cell concentration as a function of time and space, is governed by the parameter $D^{*}$. Given that $L$ is constant value, it becomes clear that the $D / \rho$ ratio is the parameter that determines the tumor cell distribution in time and space.

In order to test the effect of the $D / \rho$ ratio on the quantity and distribution of subthreshold tumor, the growth of nine tumors with $D / \rho$ ratios ranging from 0.1 to 2.0 was simulated using the traditional 1D isotropic tumor growth model, and also simulated with incorporation of DTI data to account for 3D anisotropic diffusion of tumor cells (Fig. 1). Previously published values for a group of $32 \mathrm{GBM}$ had an average D-value of 0.08 and an average p-value of 0.09 , and average $D / \rho$ ratios well within the range tested in this study (8). The DTI data used for the simulations was obtained from a healthy volunteer, and used uniformly for all simulations. For consistency, each simulated tumor in the 3D simulation had an identical starting location in the left parietal centrum semiovale. Also for consistency, each tumor was grown until its post-contrast $\mathrm{T} 1$ radius reached $1.5 \mathrm{~cm}$. For each tumor, the corresponding $\mathrm{T} 2$ radius as well as the percent of tumor cells beyond the $\mathrm{T} 2$ radius was also calculated. In order to calculate the $\mathrm{T} 1$ radius of the simulated tumors, the $\mathrm{T} 1$ boundary was conceptualized as an isocline of tumor cell concentration, such that any location in which the concentration of tumor cells was above a certain threshold, namely $80 \%$ of the tumor cell carrying capacity of brain tissue, was assumed to be visible on contrast-enhanced $\mathrm{T} 1$ imaging as enhancing tumor. Calculation of the $\mathrm{T} 2$ radius was analogous, except that the $\mathrm{T} 2$ threshold was set at $16 \%$ of the tumor cell carrying capacity of brain tissue. These values for the contrast-enhanced $\mathrm{T} 1$ and $\mathrm{T} 2$ thresholds have been previously used in several studies and are based on the correlation between histopathological calculations of tumor cell concentration and observed glioblastoma radii on MR imaging $(6,24,25)$. In calculating the farthest extent of tumor cells beyond the $\mathrm{T} 2$ radius, the farthest location from the center of the tumor in which tumor cell concentration was $>10$ cells $/ \mathrm{mm}^{3}$ was calculated in both the 1D isotropic model and the 3D anisotropic DTI model. This cut-off was used as opposed to the point at which tumor cell concentration dropped to zero. The reason for this is that the highly infiltrative nature of GBM leads to diffuse spread of tumor cells such that tumor cells, albeit at very low concentrations of $\sim 1$ cell $/ \mathrm{mm}^{3}$, may be found nearly throughout the brain within days of the onset of tumor growth regardless of 
A

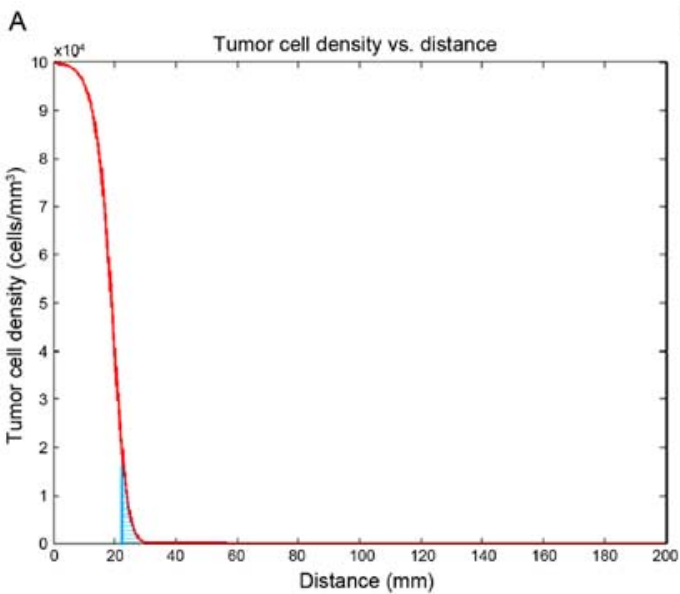

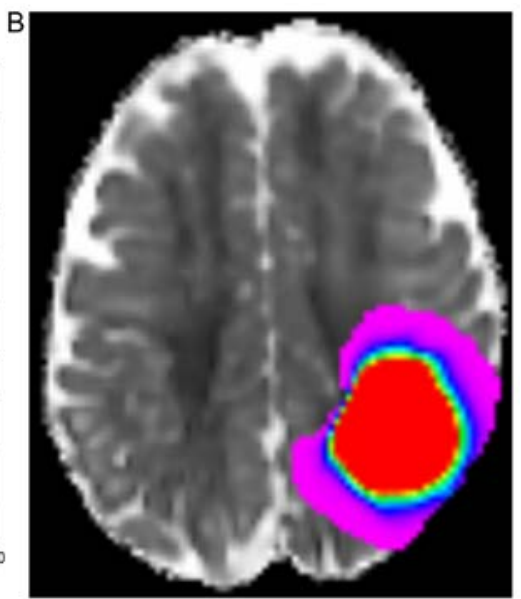

Figure 1. (A) Simulation of one-dimensional (1D) isotropic GBM growth for a tumor with $D / \rho=2.0$. The shaded region in blue represents the subthreshold tumor. (B) Simulation of three-dimensional (3D) anisotropic GBM growth for a tumor with $D / \rho=2.0$. The extent of the subthreshold tumor is represented by the magenta zone.

the relative rates of cellular diffusion and proliferation, as per the DTI model simulations. Interestingly, this matches with histologic GBM data in rats, where GBM cells could be found throughout the CNS within days of implantation (26-28). Thus, using 10 cells $/ \mathrm{mm}^{3}$ as a minimum threshold provided a more clinically relevant assessment of tumor invasion that could be used to discriminate between tumors with different growth kinetics.

This initial investigation was extended to consider the effect of the initial tumor location in the anisotropic model on the percent and extent of tumor cells beyond the T2 boundary. While the isotropic growth model leads to the production of a spherically symmetrical tumor mass regardless of the theoretical starting location of the tumor, the shape of the tumor in the anisotropic model depends on the relative rates of diffusion in the local environment of the initial tumor cells. Therefore, tumors with the same $D / \rho$ ratio may display different patterns of growth depending on where in the brain the tumor originates. To investigate this possibility, we simulated the growth of a tumor with $D / \rho=2$ in the left parietal centrum semiovale. The tumor was grown until its $\mathrm{T} 1$ radius was $1.5 \mathrm{~cm}$ and the number of days required to reach this radius was recorded. A second tumor with $D / \rho=2$ was grown for the same number of days in the corpus callosum. This was carried out in order to assess the effect on initial tumor location on tumor growth and, in particular, on the percent and extent of tumor cells beyond the T2 threshold.

Finally, the anisotropic growth model was applied to an actual patient in order to demonstrate how this model allows for clinically applicable and patient-specific simulation of tumor growth and characterization of the subthreshold tumor. In order to do this, MR imaging from two different time points was used to calculate a radial velocity based on the change in enhanced tumor radius between the two imaging time points. It has previously been established that the radial velocity of glioblastoma growth can be reasonably described by an equation known as the Fisher approximation, which states that $v=2 \sqrt{D \rho}$, where $v$ is the radial velocity of tumor growth $(8,13,24)$. As outlined previously by several investigators, the Fisher approximation enables use of the patient-specific radial velocity calculated from serial MR imaging to calculate the unique values of $D$ and $\rho$ for each patient's tumor $(6,8,24)$.

MRI methods. Imaging was performed on a Siemens 3T Magnetom scanner, with standard T1 (TR $487 \mathrm{msec}$, TE $16 \mathrm{msec}$, 5-mm slice thickness) and T2 (TR 3,670 msec, TE $93 \mathrm{msec}, 5-\mathrm{mm}$ slice thickness). DTI imaging was performed using a $128 \times 128$ matrix with a voxel size of $1.7 \times 1.7 \times 4 \mathrm{~mm}$, TR 4,100 msec, TE $95 \mathrm{msec}$, using a bipolar diffusion schema with 64 non-colinear imaging directions, $\mathrm{b}=0$ and $1,000 \mathrm{sec} / \mathrm{mm}^{2}$, $\mathrm{NEX}=3$, with 30 contiguous slices covering the entire brain.

\section{Results}

For the nine simulated tumors with $D / \rho$ ranging from 0.1 to 2.0 , higher values of $D / \rho$ corresponded to higher proportions of the subthreshold tumor in both the 1D isotropic and 3D anisotropic growth models. Furthermore, the anisotropic growth model predicted a higher percentage of tumor cells beyond the T2 threshold than did the isotropic model. As an example of these two results, isotropic modeling of the tumor with $D / \rho=0.1$ demonstrated $1.9 \%$ of tumor cells beyond the T2 threshold whereas anisotropic modeling suggested that $2.8 \%$ of tumor cells lied beyond the T2 threshold. In comparison, isotropic growth of a tumor with $D / \rho=2.0$ showed $7.6 \%$ of tumor cells beyond the T2 threshold whereas anisotropic modeling suggested that $8.9 \%$ of the tumor cells were invisible on standard MR imaging (Fig. 2). Results for the isotropic and anisotropic growth of the nine simulated tumors are illustrated in Fig. 3A.

Similar to the trends observed for the proportion of the subthreshold tumor, higher $D / \rho$ ratios also corresponded with a greater spatial extent of tumor cells beyond the visible T2 boundary. For the 1D isotropic growth model, the spatial extent beyond the T2 boundary ranged from $4 \mathrm{~mm}$ when $D / \rho$ was set at 0.1 , to $14 \mathrm{~mm}$ for $D / \rho=2.0$. Just as for the proportion of the subthreshold tumor, the 3D anisotropic growth model predicted a further spatial extent of tumor cells beyond the T2 radius than the isotropic growth model. The minimum value, 

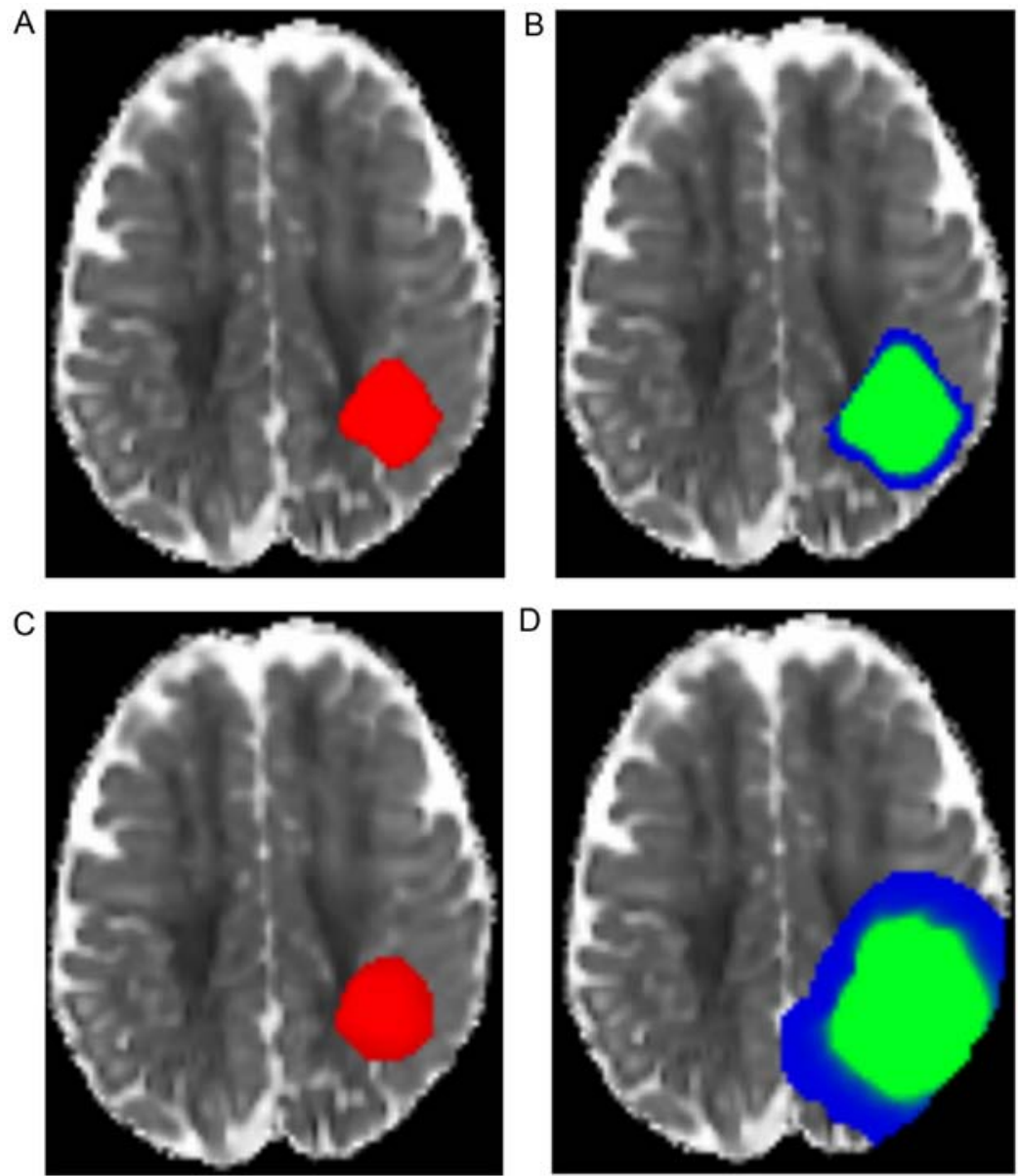

Figure 2. (A) The T1 boundary for the simulated tumor with $D / \rho=0.1$. (B) For $D / \rho=0.1$, the green region represents the visible $\mathrm{T} 2$ boundary whereas the blue region represents the subthreshold tumor. A total of $2.8 \%$ of tumor cells lie in the subthreshold region with a maximum extent of $12 \mathrm{~mm}$ beyond the $\mathrm{T} 2$ boundary. (C) $\mathrm{T} 1$ boundary for the simulated tumor with $D / \rho=2.0$. (D) For $D / p=2.0$ the green region represents the visible $\mathrm{T} 2$ boundary whereas the blue represents the subthreshold tumor. A total of $8.9 \%$ of tumor cells lie in the subthreshold region with a maximum extent of $25 \mathrm{~mm}$ beyond the T2 boundary.
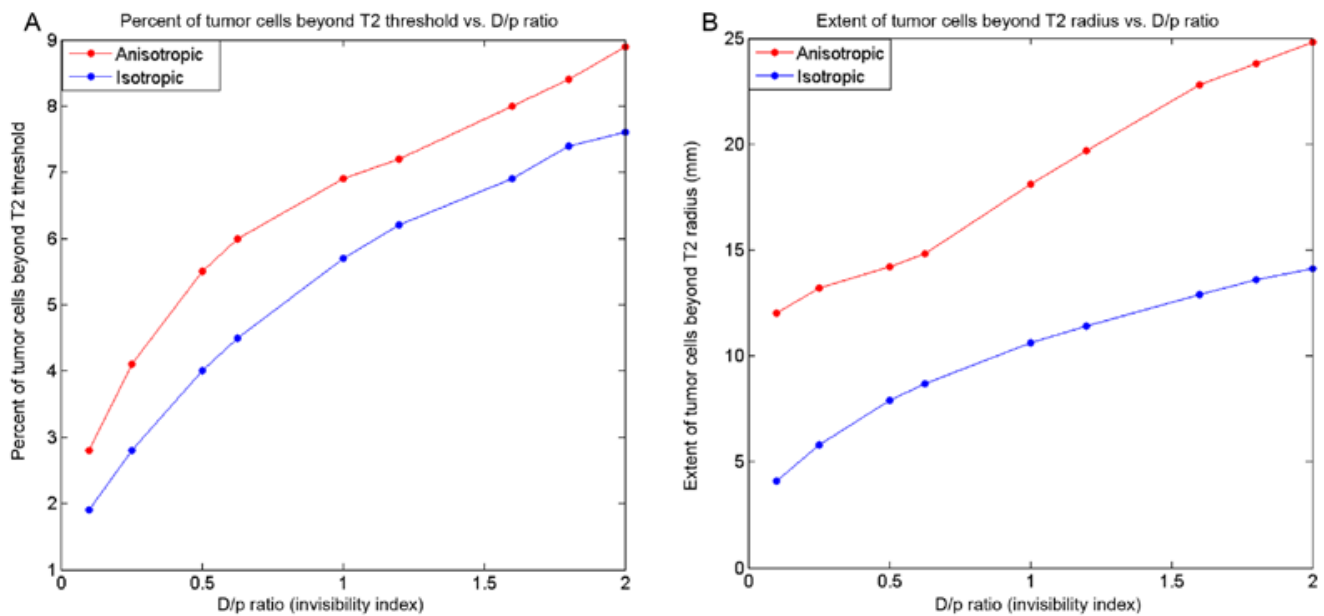

Figure 3. (A) Comparison of one-dimensional (1D) isotropic and three-dimensional (3D) anisotropic calculation of the percent of tumor cells below the T2 threshold for a $D / \rho$ range from 0.1 to 2.0. (B) Comparison of isotropic and anisotropic calculation of the maximum extent (in millimeters) of tumor cells beyond the visible $\mathrm{T} 2$ boundary.

with $D / \rho=0.1$, was $12 \mathrm{~mm}$ whereas the maximum value, when $D / \rho=2.0$, was $25 \mathrm{~mm}$ beyond the T2 radius. Full results for the nine simulated tumors are illustrated in Fig. 3B.

With the $D / \rho$ ratio was kept constant at 2.0 , tumor growth was simulated in two different locations: the corpus callosum and the left parietal centrum semiovale. As noted above, anisotropic modeling of the tumor centered in the left parietal centrum semiovale demonstrated that $8.9 \%$ of tumor cells fell below the T2 threshold and that the subthreshold tumor extended $25 \mathrm{~mm}$ beyond the T2 radius. In contrast, $8.0 \%$ of 
tumor cells were subthreshold for the tumor centered in the corpus callosum with a maximum extent of $21 \mathrm{~mm}$ beyond the T2 boundary (Fig. 4). Thus, while the percent of tumor cells beyond the $\mathrm{T} 2$ boundary was similar for the two tumors, there was greater variability in the maximum extent of the subthreshold tumor.

Finally, we applied the anisotropic growth model to a patient diagnosed with GBM in the left parietal lobe. Serial MR imaging revealed an increase of $4.0 \mathrm{~mm}$ in the contrast-enhanced $\mathrm{T} 1$ radius of the tumor from 9.1 to $13.1 \mathrm{~mm}$ over the course of 30 days, corresponding to a radial tumor growth velocity of $0.13 \mathrm{~mm} /$ day. This radial velocity yielded patient-specific rates of tumor diffusion and proliferation of $0.0825 \mathrm{~mm}^{2} /$ day and $0.33 /$ day respectively. These rates were applied to the anisotropic growth model and the simulated tumor was grown to a $\mathrm{T} 1$ radius matching that from the second day of observation. Based on the specific growth kinetics of this patient's tumor as well as the initial location of this patient's tumor, the anisotropic model suggests that $5.1 \%$ of tumor cells fell below the T2 threshold and that the subthreshold tumor extended $11.9 \mathrm{~mm}$ beyond the visible T2 margin (Fig. 5).

\section{Discussion}

The combination of serial MR imaging and mathematical modeling represents an innovative and important approach to individually tailored simulation of glioblastoma growth on a patient-by-patient basis $(6,8,9)$. The reaction-diffusion partial differential equation model presented above accounts for both the proliferative and invasive properties of GBM that, taken together, lead to poor prognoses with relatively short survival times. The relative rates of tumor cell diffusion and proliferation, i.e., the $D / \rho$ ratio or invisibility index of the tumor, correlate to the proportion of cells beyond the visible T2/FLAIR margin (6). Additionally, it was shown that the $D / \rho$ ratio was correlated to the maximum spatial extent of tumor cells beyond the T2 boundary such that tumors with higher $D / \rho$ ratios extended farther beyond visible tumor margins. This correlation may significantly affect the outcome of radiation therapy or surgical resection. To this end, it has already been preliminarily demonstrated that the $D / \rho$ ratio, used in the context of the above model and incorporating the extent of surgical resection, aids in the accurate prediction of survival of GBM patients (10).

Hence, this sort of modeling has the potential to offer clinically significant information. Initial modeling efforts considered the brain to be a 1D homogenous mass, through which tumor cells diffuse at a rate independent of their location $(6-8,12,13)$. While subsequent studies have recognized the increased diffusion of tumor cells in white matter, it is only the most recent research that has begun to incorporate DTI data in order to model anisotropic tumor growth $(15,21)$. The advantages of the anisotropic tumor growth model are several. Firstly, simulations of tumor growth incorporating DTI data have already demonstrated a better empirical fit to actual tumor progression as observed on MR imaging. One study focused on using DTI data to model the growth of low-grade gliomas concluded that the observed tumor shapes could only be reproduced by simulation with the anisotropic growth model. In contrast, the isotropic growth models, even those accounting for differing rates of diffusion in white and gray matter, could not adequately reproduce the shape of a patient's tumor (21). In this study, we corroborated that result by applying the anisotropic growth model to a patient with glioblastoma in the left parietal lobe. Comparison of the T1 boundary of the simulated tumor to that of the actual patient demonstrated that the $3 \mathrm{D}$ anisotropic model more closely resembled the patient's tumor than the isotropic model would, which would simply produce a spherical tumor in three dimensions (Fig. 5). Thus, the close correspondence between observed tumor growth and the results of the anisotropic growth model suggest an improved accuracy of the anisotropic model over the isotropic model.

The ability of the anisotropic model to more accurately predict the cellular distribution of the visible portion of tumor on MR imaging supports the notion that this model can also more accurately predict the quantity and spatial distribution of the subthreshold tumor. Thus, this study describes two significant results with respect to the subthreshold component of GBM. Firstly, higher $D / \rho$ ratios lead to an increased proportion of tumor cells as well as a greater spatial distribution of tumor cells beyond the $\mathrm{T} 2$ radius - a finding that held for both the isotropic and anisotropic growth models. Secondly, although the isotropic and anisotropic models demonstrated similar trends of greater subthreshold tumor for greater $D / \rho$ ratios, the anisotropic model consistently and significantly predicted a higher proportion and greater spread of tumor cells beyond the T2 boundary for any given $D / \rho$ ratio.

These results are of great clinical importance. Despite the overall modest response of GBM to treatment with surgery, chemotherapy, and radiotherapy, radiotherapy has been shown to be the most effective of the three treatment modalities with regards to improving patient prognosis (29). Therefore, the ability to individualize and optimize radiation plans provides an important avenue for achieving optimal patient outcomes. As mentioned above, radiation fields typically employ margins of $1-3 \mathrm{~cm}$ beyond the region of observable tumor on imaging (5). Given the dimensions of the brain, this wide range of clinically acceptable margin underscores the very large degree of variability in possible radiation fields. It is in this context that accurate characterization of the subthreshold tumor can provide important information for determining the shape and size of optimal radiation fields designed to deliver large doses of radiation to a tumor while sparing, as much as possible, normal brain tissue from potentially harmful exposure to radiation. For example, the tumor simulated in this study with $D / \rho=0.1$ had a greatest cellular extent of $12 \mathrm{~mm}$ beyond the $\mathrm{T} 2$ radius. Thus, for tumors with low $D / \rho$ such as this, a $3 \mathrm{~cm}$ margin may lead to excessive radiation of brain tissue containing an exceedingly small concentration of tumor cells. Such a tumor may theoretically benefit from larger radiation doses confined to a smaller field. Contrarily, tumors with larger $D / \rho$ ratios, such as the simulated tumor with $D / \rho=2.0$, may have an extent of tumor cells $>2.5 \mathrm{~cm}$ beyond the $\mathrm{T} 2$ radius. For such tumors, radiation fields with a margin of 1 or $2 \mathrm{~cm}$ would not adequately treat the tumor. Furthermore, the anisotropic model serves as a better clinical tool than the 1D isotropic model for assessing the trade-off between establishing larger margins at the cost of delivering higher doses to normal brain tissue as opposed to establishing smaller margins at the 
A

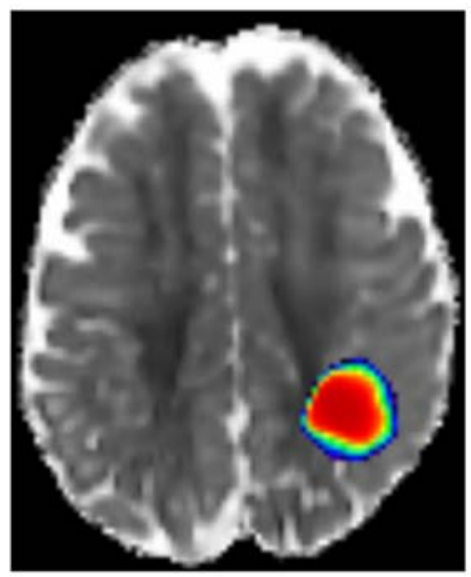

C

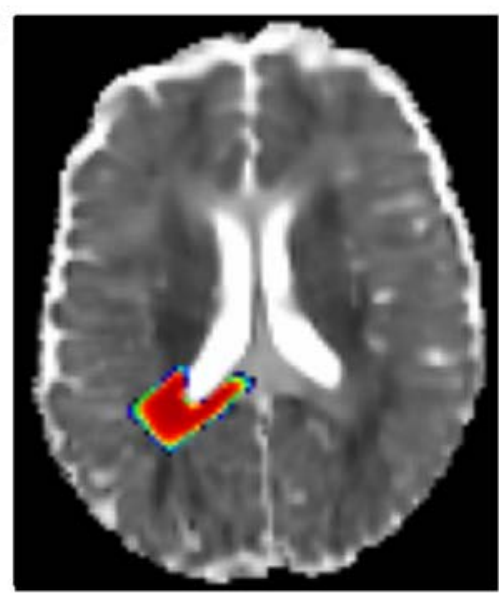

B
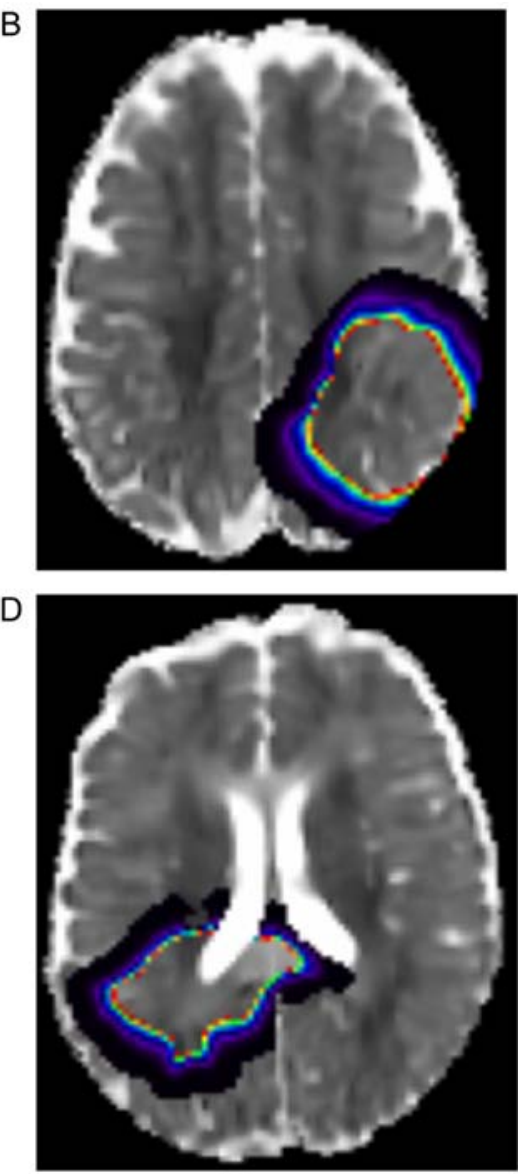

Figure 4. (A) Simulated T1 radius for a tumor with $D / \rho$ ratio=2.0 centered in the left parietal centrum semiovale. (B) Corresponding subthreshold tumor, containing $8.9 \%$ of tumor cells with a maximum extent of $25 \mathrm{~mm}$ beyond the T2 radius. (C) Simulated T1 radius for a tumor with $D / \rho=2.0$ centered in the corpus callosum. (D) Corresponding subthreshold tumor, containing $8.0 \%$ of tumor cells with a maximum extent of 21 mm beyond the T2 radius. It is noted that the extent and the shape of the zone of the subthreshold tumor critically depends on the tumor's location. This reflects a significant contribution of the DTI model, potentially allowing the design of treatment fields to reflect expected tumor infiltration along local white matter tracts.
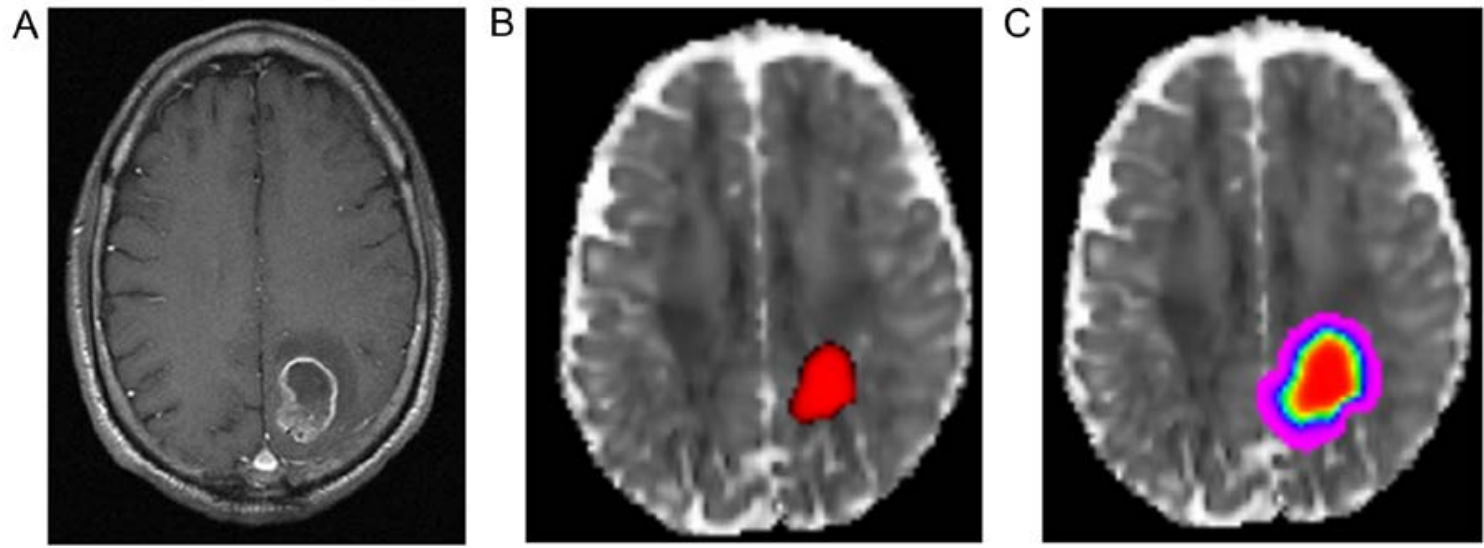

Figure 5. (A) Observed T1 weighted MRI for a patient with GBM in the left parietal lobe. Values of $D$ and $\rho$ were calculated for this patient to be $0.0825 \mathrm{~mm}^{2} / \mathrm{day}$ and 0.33 /day respectively. (B) Anistropically simulated T1 boundary for GBM with equivalent values of $D$ and $\rho$. (C) For the simulated tumor, the T1 region is shown in red while the extent of the subthreshold tumor is shown in pink. The relatively small zone of subthreshold tumor infiltration in this patient reflects the low $D / \rho$ ratio, and illustrates the direct correlation between the size of this zone and the magnitude of that ratio.

cost of failing to deliver radiation to the tumor cells beyond the treatment margins. For example, 1D isotropic modeling of the tumor with $D / \rho=2.0$ suggests that margins of $1.5 \mathrm{~cm}$ would deliver radiation to the entire tumor mass. However, the more accurate $3 \mathrm{D}$ anisotropic modeling demonstrates that tumor cells extend farther than $2 \mathrm{~cm}$ beyond the T2 radius. Thus, the more accurate characterization of the subthreshold tumor offered by the anisotropic growth model allows for more informed clinical decisions with regards to radiation therapy planning. 
This point is of particular importance given recent efforts to find optimal treatment schemes to improve patient survival while at the same time decreasing aggregate dose to normal brain tissue. One such study using an isotropic growth model determined that optimal dosing schemes delivered spatially variable dose within the radiation field in a manner determined by the spatial distribution of tumor cells within the field, as dictated by the patient-specific $D / \rho$ ratio (24). This result implies that true optimization of radiation therapy plans depends on the ability to accurately use the patient-specific $D / \rho$ ratio to describe tumor cell concentration in space. Because the use of DTI data to model anisotropic tumor growth allows for more accurate modeling of tumor cell concentration based on the $D / \rho$ ratio, anisotropic tumor modeling represents an important advancement in efforts to optimize radiation therapy protocols.

The true clinical value of the reaction-diffusion model for glioblastoma growth lies in the potential to personalize this model to each patient's tumor. Traditionally, individualization of the model to each patient entails the calculation of tumor-specific values of $D$ and $\rho$ to describe the rates of diffusion and proliferation particular to each patient. However, the implementation of anisotropic modeling in this study suggests that tumors with the same values of $D$ and $\rho$, and thus the same $D / \rho$ ratio, will display different growth patterns and have different proportions and spatial distribution of the subthreshold tumor depending on the initial location of tumor growth. In this way, incorporation of DTI data to model anisotropic tumor growth does more than simply improve the accuracy of already established methods; rather it reframes the notion of personalized tumor modeling to designate initial tumor location as a personalized modeling parameter alongside the already established individualized values of $D$ and $\rho$.

Clearly, mathematical models are idealizations of a much more complex underlying biology, but they have utility if they can capture essential features of the phenomenon under study. Nevertheless, this model has several limitations. In general, of course, there is cellular heterogeneity within all tumors, and hence calculation of parameters such as $D$ and $\rho$ represents an aggregate measure. Furthermore, precise values of $D$ and $\rho$ may vary temporally, as the tumor de-differentiates over time. Another limitation is that invasiveness is an active process, and can only be roughly modeled as a passive diffusion phenomenon. A significant limitation also has to do with the cell-density thresholds set up for tumor visibility on contrast-enhanced T1 and on T2 weighted images. At present, these are only rough approximations, and would certainly be expected to have some variations across different tumors, based on the extent of neovascularity and the extent of breakdown of the blood-brain barrier. With regards to DTI data, the present study does not account for possible variability in the diffusion tensor due to mass effect of the tumor as it grows. Therefore, the quantitative data generated by the model are of necessity inexact. However, they can be regarded as giving some important qualitative insights regarding expected behavior of tumors based on their $D / \rho$ ratio, and can serve as a rough guide in adjusting radiation fields or surgical margins.

In conclusion, since its initial use, the reaction-diffusion model for glioma growth has evolved with regards to its primary applications. While the model was initially used to simulate untreated glioma growth, subsequent studies have incorporated the effects of radiation therapy to simulate tumor growth with standard radiotherapy protocols. Still more recent studies have sought to use the extended radiation therapy model to search for optimized radiotherapy regimens with the finding that optimal treatment depends on the particular $D / \rho$ ratio for each patient's tumor - as it is the $D / \rho$ ratio that dictates the spatial distribution and cell concentration of tumors even beyond the observable tumor boundaries on MR imaging. Simultaneous with this evolution of application, the reaction-diffusion model has also evolved with regards to implementation. Initial studies modeled GBM growth in one spatial dimension and conceived of the diffusion coefficient, $D$, as a single constant value applicable throughout the brain. While later studies assigned one value of $D$ for diffusion of tumor cells through gray matter and a separate value of $D$ for diffusion in white matter, it is only the most recent studies that have begun implementing DTI as a means of modeling $3 \mathrm{D}$ anisotropic glioma growth. With its ability to account for anatomical boundaries, increased diffusion of tumor cells in white matter, and increased diffusion of tumor cells along the direction of white matter tracts, DTI represents the most sophisticated and accurate implementation of the reaction-diffusion model to date. It is in this context, with regards to both application and implementation, that the current study seeks to use anisotropic modeling of glioblastoma growth to examine the effects of different $D / \rho$ ratios and different initial tumor location on the quantity and distribution of the subthreshold tumor. This is important, as accurate characterization of the subthreshold tumor has direct implications on the establishment of radiation field margins. Furthermore, the tumor cell distribution, as dictated by the invisibility index, has direct applications to the optimization of dose distribution within the chosen radiation field.

\section{Acknowledgements}

No ethical approval was required since this article does not contain any studies with human participants or animals performed by any of the authors.

\section{References}

1. Salcman M: Survival in glioblastoma: Historical perspective. Neurosurgery 7: 435-439, 1980.

2. Salford LG, Brun A and Nirfalk S: Ten-year survival among patients with supratentorial astrocytomas grade III and IV. J Neurosurg 69: 506-509, 1988.

3. Lassman AB and Holland EC: Glioblastoma multiforme - past, present and future. US Oncol Rev 1: 109-111, 2005.

4. Stupp R, Mason WP, van den Bent MJ, Weller M, Fisher B, Taphoorn MJ, Belanger $\mathrm{K}$, Brandes AA, Marosi C, Bogdahn U, et al; European Organisation for Research and Treatment of Cancer Brain Tumor and Radiotherapy Groups; National Cancer Institute of Canada Clinical Trials Group: Radiotherapy plus concomitant and adjuvant temozolomide for glioblastoma. N Engl J Med 352: 987-996, 2005.

5. Becker KP and Yu J: Status quo - standard-of-care medical and radiation therapy for glioblastoma. Cancer J 18: 12-19, 2012.

6. Harpold HL, Alvord EC Jr and Swanson KR: The evolution of mathematical modeling of glioma proliferation and invasion. J Neuropathol Exp Neurol 66: 1-9, 2007.

7. Tracqui P, Cruywagen GC, Woodward DE, Bartoo GT, Murray JD and Alvord EC Jr: A mathematical model of glioma growth: The effect of chemotherapy on spatio-temporal growth. Cell Prolif 28: 17-31, 1995. 
8. Wang CH, Rockhill JK, Mrugala M, Peacock DL, Lai A, Jusenius K, Wardlaw JM, Cloughesy T, Spence AM, Rockne R, et al: Prognostic significance of growth kinetics in newly diagnosed glioblastomas revealed by combining serial imaging with a novel biomathematical model. Cancer Res 69 : 9133-9140, 2009.

9. Murray JD: Growth and control of brain tumors. In: Mathematical Biology II: Spatial Models and Biomedical Applications. Antman SS, Marsden JE, Sirovich L and Wiggins S (eds). Vol 18. 3rd edition. Springer, New York, NY, pp536-613, 2003.

10. Swanson KR, Rostomily RC and Alvord EC Jr: A mathematical modelling tool for predicting survival of individual patients following resection of glioblastoma: A proof of principle. $\mathrm{Br} \mathbf{J}$ Cancer 98: 113-119, 2008 .

11. Woodward DE, Cook J, Tracqui P, Cruywagen GC, Murray JD and Alvord EC Jr: A mathematical model of glioma growth: The effect of extent of surgical resection. Cell Prolif 29: 269-288, 1996.

12. Rockne R, Alvord EC Jr, Rockhill JK and Swanson KR: A mathematical model for brain tumor response to radiation therapy. J Math Biol 58: 561-578, 2009.

13. Rockne R, Rockhill JK, Mrugala M, Spence AM, Kalet I, Hendrickson K, Lai A, Cloughesy T, Alvord EC Jr and Swanson KR: Predicting the efficacy of radiotherapy in individual glioblastoma patients in vivo: A mathematical modeling approach. Phys Med Biol 55: 3271-3285, 2010.

14. Burgess PK, Kulesa PM, Murray JD and Alvord EC Jr: The interaction of growth rates and diffusion coefficients in a three-dimensional mathematical model of gliomas. J Neuropathol Exp Neurol 56: 704-713, 1997.

15. Swanson KR, Alvord EC Jr and Murray JD: A quantitative model for differential motility of gliomas in grey and white matter. Cel Prolif 33: 317-329, 2000.

16. Beliën AT, Paganetti PA and Schwab ME: Membrane-type 1 matrix metalloprotease (MT1-MMP) enables invasive migration of glioma cells in central nervous system white matter. J Cell Biol 144: 373-384, 1999.

17. Giese A and Westphal M: Glioma invasion in the central nervous system. Neurosurgery 39: 235-250, discussion 250-252, 1996.

18. Giese A, Bjerkvig R, Berens ME and Westphal M: Cost of migration: Invasion of malignant gliomas and implications for treatment. J Clin Oncol 21: 1624-1636, 2003.
19. Pedersen PH, Edvardsen K, Garcia-Cabrera I, Mahesparan R, Thorsen J, Mathisen B, Rosenblum ML and Bjerkvig R: Migratory patterns of lac-z transfected human glioma cells in the rat brain. Int J Cancer 62: 767-771, 1995.

20. Yoshida D, Watanabe K, Noha M, Takahashi H, Teramoto A and Sugisaki Y: Tracking cell invasion of human glioma cells and suppression by anti-matrix metalloproteinase agent in rodent brain-slice model. Brain Tumor Pathol 19: 69-76, 2002.

21. Jbabdi S, Mandonnet E, Duffau H, Capelle L, Swanson KR, Pélégrini-Issac M, Guillevin R and Benali H: Simulation of anisotropic growth of low-grade gliomas using diffusion tensor imaging. Magn Reson Med 54: 616-624, 2005.

22. Herculano-Houzel S and Lent R: Isotropic fractionator: A simple, rapid method for the quantification of total cell and neuron numbers in the brain. J Neurosci 25: 2518-2521, 2005.

23. Basser PJ, Mattiello J and LeBihan D: MR diffusion tensor spectroscopy and imaging. Biophys J 66: 259-267, 1994.

24. Corwin D, Holdsworth C, Rockne RC, Trister AD, Mrugala MM, Rockhill JK, Stewart RD, Phillips M and Swanson KR: Toward patient-specific, biologically optimized radiation therapy plans for the treatment of glioblastoma. PLoS One 8: e79115, 2013.

25. Swanson KR and Alvord EC Jr: Serial imaging observations and postmortem examination of an untreated glioblastoma: A traveling wave of glioma growth and invasion. Neuro-oncol 4: $340,2002$.

26. Silbergeld DL and Chicoine MR: Isolation and characterization of human malignant glioma cells from histologically normal brain. J Neurosurg 86: 525-531, 1997.

27. Chicoine MR and Silbergeld DL: Assessment of brain tumor cell motility in vivo and in vitro. J Neurosurg 82: 615-622, 1995.

28. Chicoine MR and Silbergeld DL: The in vitro motility of human gliomas increases with increasing grade of malignancy. Cancer 75: 2904-2909, 1995.

29. Unkelbach J, Menze BH, Konukoglu E, Dittmann F, Le M, Ayache $\mathrm{N}$ and Shih HA: Radiotherapy planning for glioblastoma based on a tumor growth model: Improving target volume delineation. Phys Med Biol 59: 747-770, 2014. 